\title{
A variant on the kappa opioid receptor gene (OPRK1) is associated with stress response and related drug craving, limbic brain activation and cocaine relapse risk
}

\author{
$\mathrm{K} \mathrm{Xu}^{1}$, D Seo ${ }^{1}, \mathrm{C}$ Hodgkinson $^{2}, \mathrm{Y} \mathrm{Hu}^{3}$, D Goldman ${ }^{2}$ and R Sinha ${ }^{1,4,5}$
}

Stress increases drug craving and relapse risk. The kappa opioid receptor gene (OPRK1) mediates stress responses. Here, we examined whether the OPRK1 rs6989250 C > G affects stress-induced cocaine craving and cortisol responses, subsequent cocaine relapse risk and the neural response to stress using functional magnetic resonance imaging (fMRI) in cocaine dependence. Sixty-seven treatment-engaged, abstinent cocaine-dependent African-Americans were genotyped (CG: $N=10 ; C C$ : $N=57$ ) and participated in a 3-day experiment in which they were exposed to personalized script-driven imagery of stress, drug cues and neutral scenarios, one condition per day, randomly assigned and counterbalanced across subjects. Repeated measures of craving and cortisol were obtained. The subjects were followed prospectively for 90 days to assess relapse risk. A follow-up preliminary fMRI experiment assessed neural responses to stress, drug cue and neutral conditions in matched CG $(N=5)$ and CC $(N=8)$ subgroups. We found greater stress-induced craving $(P=0.019)$, higher cortisol during stress and cue relative to the neutral condition ( $\left.P^{\prime} \mathbf{S}<0.003\right)$, and increased cocaine relapse risk $(P=0.0075)$ in the CG compared with the CC group. The CG relative to the CC group also showed greater activation of limbic and midbrain regions during stress and cues relative to the neutral condition with additional stress-induced activation in the right amygdala/hippocampus $(P<0.05$, wholebrain corrected). These results suggest that OPRK1 is associated with stress-induced craving and cortisol, hyperactive hypothalamus/thalamus-midbrain-cerebellum responses, and also associated with greater subsequent cocaine relapse risk. Future studies to replicate these findings in a larger sample size are warranted.

Translational Psychiatry (2013) 3, e292; doi:10.1038/tp.2013.62; published online 20 August 2013

\section{Introduction}

Relapse to drug use is the central problem in the treatment of addiction. ${ }^{1}$ It is well known that acute and chronic stress increases drug craving and also contributes to high rates of addiction relapse. ${ }^{2,3}$ However, the remarkable individual variation observed in stress and drug craving suggests that genetic variants may contribute to the differences in response to stress, craving and relapse risk.

Converging evidence shows that the kappa opioid receptor (KOR) has an important role in mediating the stress response and in stress-related addictive processes. ${ }^{4-8}$ Activation of the KOR through the administration of dynorphin, a KOR endogenous neuropeptide, produces stress aversive symptoms in rats. ${ }^{6,7,9,10}$ Physical stress and social defeat elevate dynorphin levels, ${ }^{11-14}$ suggesting that activation of the KOR system increases both the physical and psychological stress responses. KOR activation also provokes stress-induced drug reward. ${ }^{15}$ The administration of a KOR agonist mimics the forced swim stress-induced potentiation of cocaine place preference. ${ }^{16}$ In healthy human subjects, the administration of a KOR agonist produces dysphoria and anhedonia, ${ }^{5,6}$ which resemble withdrawal 'crash' symptoms in cocaine-dependent patients. Clinically, stress and dysphoria are closely associated with craving and relapse risk..$^{2,17-20}$ Genetic variants of the prodynorphin gene are associated with negative emotions and alcohol dependence. ${ }^{21}$ Thus, genetic variants of OPRK1 may modulate individual stress responses, stressinduced craving and the relapse risk associated with cocaine dependence.

KOR has significant effects on the brain regions involved in addiction. ${ }^{15,22}$ Repeated administration of a KOR agonist can increase dopamine release and cocaine-induced locomotor activity, akin to cross-sensitization of stress and drug reward. ${ }^{23}$ Dynorphin mRNA expression and KOR binding are elevated in dopamine-rich regions in the post-mortem brain tissue of cocaine-dependent patients. ${ }^{24}$ These findings suggest that the activation of KOR produces drug reward effects by increasing dopamine release. Thus, it is possible that KOR may affect stress-related addictive phenotypes, such as craving and relapse.

\footnotetext{
${ }^{1}$ Department of Psychiatry, Yale School of Medicine, New Haven, CT, USA; ${ }^{2}$ Laboratory of Neurogenetics, National Institute on Alcohol Abuse and Alcoholism, Bethesda, MD, USA; ${ }^{3}$ Center for Biomedical Informatics and Information Tech, National Cancer Institute, Bethesda, MD, USA; ${ }^{4}$ Child Study Center, Yale School of Medicine, New Haven, CT, USA and ${ }^{5}$ Stress Center, Yale University, New Haven, CT, USA

Correspondence: Professor R Sinha, Yale University School of Medicine, 2 Church Street South, Suite 209, New Haven, CT 06519, USA.

E-mail: rajita.sinha@yale.edu

Keywords: cocaine craving; limbic-midbrain hyperactivation; OPRK1; relapse; stress

Received 18 March 2013; revised 31 May 2013; accepted 4 June 2013
} 
Genetic variants of OPRK1 have previously been linked to substance dependence with inconsistent results. ${ }^{8,21,25-28}$ One single-nucleotide polymorphism (SNP) in intron 2 of OPRK1 has been associated with alcohol dependence, ${ }^{25}$ whereas three SNPs in the coding region show no association with this condition. ${ }^{29}$ The frequency of OPRK1 haplotypes with intronic SNPs was found to be higher in alcoholdependent subjects compared with controls. ${ }^{26}$ A recent study found that the non-coding SNP rs997917 was associated with alcohol-induced sedation. ${ }^{30}$ Furthermore, an SNP in a noncoding region was associated with opioid dependence in a Caucasian population. ${ }^{27}$ These data suggest that the regulatory region of OPRK1 may be important for substance dependence. In support of this notion, a promoter SNP was identified in alcohol-avoiding rats, ${ }^{31}$ and a promoter 800 -bp indel variant of OPRK1 was found to affect gene expression in vitro. ${ }^{32}$ The longer allele showed $50 \%$ lower expression than the shorter allele. Interestingly, this functional promoter variant has been associated with alcohol dependence in families. ${ }^{32}$ However, no previous research has assessed the effects of OPRK1 variants and stress, drug craving and relapse risk in a cocaine-dependent population.

In this study, we examined whether the SNP rs6989250 $\mathrm{C}>\mathrm{G}$ on the $5^{\prime}$-regulatory region of OPRK1 affected stressinduced cocaine craving and cortisol in the laboratory and subsequent cocaine relapse risk. We focused on this particular SNP because: (1) it is located in the $5^{\prime}$-regulatory region of $O P R K 1$, and is close to the functional indel variant described above and (2) the rare $\mathrm{G}$ allele creates an NF-kB transcription binding site, suggesting a potential alteration of OPRK1 function. Because $\mathrm{G}$ allele at rs6989250 is rare in Caucasians but abundant in Africans (http://hapmap. ncbi.nlm.nih.gov/cgi-perl/gbrowse/hapmap28_B36/\#search), we tested the association of rs6989250 with stress, drug craving and relapse risk in an African-American sample of cocaine-dependent patients. As greater stress reactivity would also suggest higher neural activity in stress-sensitive limbic regions, such as the hypothalamus, amygdala, and hippocampus, we conducted a preliminary follow-up functional magnetic resonance imaging (fMRI) study to explore whether rs6989250 is associated with neural activation in the stress-responsive brain circuits of cocaine-dependent subjects.

\section{Materials and methods}

Participants. Male and female subjects between the ages of 21 and 50 seeking inpatient treatment for cocaine dependence were evaluated for study participation (male: $N=36$; female: $N=31$ ). Subjects who met the Diagnostic and Statistical Manual of Mental Disorders (DSM-IV) criteria for current cocaine dependence were admitted to the CNRU (Clinical Neuroscience Research Unit) of the CMHC (Connecticut Mental Health Center) for 2-4 weeks of inpatient treatment and research participation in a three-session laboratory experiment. Individuals who met the DSM-IV criteria for current dependence on additional psychoactive substances other than alcohol and nicotine were excluded. In addition, individuals on medications for medical or psychiatric diseases and those in need of alcohol detoxification were excluded from the study. The study procedures were approved by the Human Investigation Committee of the Yale University School of Medicine, and all subjects provided written informed consent.

Procedures. During their first week of being an inpatient at the CNRU, the subjects were interviewed using the Structured Clinical Interview for DSM-IV (SCID-I) ${ }^{33}$ to determine psychiatric diagnoses. Baseline demographics and drug use history were also assessed. During the second week, the subjects participated in personalized script driven guided imagery procedures as outlined in previous work. . $^{2,3,34-36}$ In an imagery script development session, each subject identified a highly stressful event from their lives (rated by the subject as an 8 or above on a 10-point Likert scale for stressfulness), a personal drug cue-related event that involved people, a place and objects related to cocaine use, and a personal neutral relaxing event. Between weeks 3 and 4 , the subjects participated in a habituation and imagery training session, followed by a 3-day laboratory experiment upon awakening from 0800 to $1030 \mathrm{~h}$, in which they were exposed to a stress, drug cue and neutral relaxing imagery provocation, one script per day, order randomized and counterbalanced across subjects, as described in our previous studies. ${ }^{3,37}$ Script exposure occurred via audiotape and the length of each taped script was $\sim 4.5 \mathrm{~min}$, with a total imagery period of $5 \mathrm{~min}$ (see Supplementary Information for details).

Laboratory measures. On each day of the experiment, cocaine craving was repeatedly assessed using a 10-point VAS (visual analog scale) that measured responses to the item 'desire for using cocaine at this moment'. Repeated blood samples were also collected for cortisol assessments and processed at the $\mathrm{YCCl}$ (Yale Center for Clinical Investigation) Core Laboratories using standard radioimmunoassay procedures. On day 1 of the experiment, $10 \mathrm{ml}$ of blood was drawn for genotyping.

Cocaine craving and plasma cortisol responses were assessed at baseline, immediately following exposure to the imagery (time point 0 ) and at repeated recovery time points every $15 \min (+15,+30,+45,+60$ and +75$)$ after exposure to imagery. All time points were utilized to assess response to brief stress, drug cue and neutral cue provocation in the laboratory.

Prospective follow-up of cocaine relapse post-inpatient treatment. Appointments were scheduled for all participants for follow-up interviews in 14, 30 and 90 days after discharge from the inpatient unit. The patients were assessed for cocaine and other drug use with the Substance Use Calendar and urine and breathalyzer samples at each appointment, as described in our previous studies ${ }^{3,18}$ to prospectively assess cocaine relapse rates for the 90-day follow-up period (see Supplementary Information for followup assessments of relapse).

OPRK1 genotyping. Genotyping was performed using the Addiction Array as described previously. ${ }^{38}$ Briefly, haplotype tags for 1350 loci from 130 candidate genes were included in 
this array. Additional 186 ancestry informative markers were included in the array to test population stratification. Population substructure was defined by applying the 186 ancestry informative markers into the software 'Structure' (http://pritch.bsd.uchicago.edu/software.html). ${ }^{39}$ Genotyping of rs6989250 was obtained from the Addiction Array based on Illumina GoldenGate Technology. The frequency of the $\mathrm{G}$ allele is $10 \%$ in African-Americans and $0 \%$ in Caucasians in our studied sample population. In all, 10 of the subjects in the study were CG heterozygous and 57 subjects were CC homozygous.

Follow-up preliminary fMRI study. A follow-up preliminary fMRI study was also conducted in a demographically matched sample of five CG and eight CC patients (see Supplementary Methods for details). The magnetic resonance images were obtained using the 3-T Siemens Trio MRI system (Siemens Medical Solutions, Erlangen, Germany) with a single-channel, standard-quadrature head coil and a T2*-sensitive gradient-recalled single shot echo-planar pulse sequence. As in our previous fMRI studies, ${ }^{36,40}$ a block design was used with six fMRI trials of personalized stress, drug cue and neutral scenarios (two separate events for each condition) that were presented in a randomized and counterbalanced order, and each script was presented via audiotape. Each trial took $5 \mathrm{~min}$, which included a 1.5-min quiet baseline period followed by a 2.5 -min imagery period ( $2 \mathrm{~min}$ of read imagery and $0.5 \mathrm{~min}$ of quiet imagery) and a $1-\mathrm{min}$ quiet recovery period. During the baseline period, the participants were asked to remain in the scanner without any mental activity. Craving ratings were collected using a 10-point verbal Likert scale before and after each trial. To normalize any residual cravings from the previous trial, participants were engaged in 2 min of progressive relaxation between each trial (see Supplement Information).

Statistics. The $\chi^{2}$ test and Student's $t$-test were applied to check for differences in the age, gender, years of education, duration and quantity of cocaine, alcohol and nicotine use between the two genotype groups (CG versus $\mathrm{CC}$ ).

Linear mixed effect models were employed for analysis of cocaine craving and cortisol responses during the laboratory experiment using the SAS software package (Version 9, 2006; SAS Institute, Cary, NC, USA). The between-subject factors for Group 2 (genotyping CG versus CC), the withinsubject factors of Condition 3 (stress, drug cues and neutral) and Time point (baseline, $0,+15,+30,+45,+60$ and +75 ) were the fixed effects, whereas Subject was the random effect factor in the model. Genetically inferred ancestry obtained from the 186 ancestry informative markers was a covariate in the model. Cohen's $d$ was used to estimate the effect size. The relationship between genotypes (CG versus $\mathrm{CC}$ ) and relapse risk was examined using Log-rank survival analysis.

fMRI analysis. Using XMedCon, ${ }^{41}$ the raw imaging data were converted from the Digital Imaging and Communication in Medicine format into the Analyze format. The first 10 images were removed from the beginning of each functional run to achieve a steady-state equilibrium between radio frequency pulsing and relaxation. The images were slice time-corrected and motion-corrected for three translational and three rotational directions; ${ }^{42}$ any trial with a linear motion exceeding $1.5 \mathrm{~mm}$ or a rotation of $>2$ was discarded. On an individual basis, a General Linear Model for each voxel in the brain volume was implemented with a regressor (time during imagery) for each trial per condition. In the General Linear Model, drift correction was included, such that drift regressors were used to remove the mean time course, linear trend, quadratic trend and cubic trend for each functional run. Due to the possibility of carryover effects from the imagery period, the recovery period was excluded from the data analysis. Each functional run was spatially smoothed using a 6-mm Gaussian kernel and normalized to generate beta maps $(3.44 \mathrm{~mm} \times 3.44 \mathrm{~mm} \times 4 \mathrm{~mm})$.

Three registrations were sequentially implemented using the Yale Biolmage Suite software package (http://www. bioimagesuite.org) to adjust for individual anatomical differences, ${ }^{43}$ the linear registration of functional data into $2 \mathrm{D}$ anatomical images, the $2 \mathrm{D}$ to $3 \mathrm{D}(1 \times 1 \times 1 \mathrm{~mm})$ linear registration and non-linear registration to a reference 3D image. The reference image used was the Colin27 Brain ${ }^{44}$ from the MNI (Montreal Neurological Institute) space.

For the second-level group analysis, Analysis of Functional Neurolmages (AFNI) software ${ }^{45}$ was utilized to run random mixed effect models. A $2 \times 3$ mixed effect model (group by condition) was conducted with Group as the between-subject fixed-effect factor; Condition (stress, drug cue and neutral) as the within-subject fixed-effect factor; and Subject as the random-effect factor. For further comparison, two-tailed $t$-tests were conducted on the mean signal change contrasts (beta). To correct for multiple comparisons, a family-wise error (FWE) rate correction was applied via a Monte Carlo Simulation using Alpha Sim in AFNI. ${ }^{46}$ To selectively isolate brain activity only specific to stress and drug components of imagery processing, the fMRI data results are presented for each contrast (stress-neutral and drug-neutral) using a withinsubject subtraction method.

\section{Results}

Demographic data and individual characteristics by OPRK1 genotype. We analyzed 67 African-American cocaine-dependent individuals genotyped for rs6989250 $(C G=10 ; C C=57)$. There were no significant differences in age, years of education, the duration and amount of cocaine and alcohol use between the two genotype groups (CG versus CC) $(P>0.05)$ (Table 1). However, the CG group included significantly more cigarette smokers than the CC group (100 versus $80 \% ; P<0.05$ ). Cigarette smoking was included as a covariate in the analyses.

Cocaine craving. We first analyzed association of each SNP on the OPRK1 with cocaine craving. Among a total of 11 SNPs of OPRK1 from the Addiction Array, the SNP, rs6989250, was the only SNP significantly associated with cocaine craving $(P=0.02$ uncorrected). There was no significant association of rs6989250 with craving after multiple test correction. However, considering that environmental 
Table 1 Demographic characteristics of the two genotype groups

\begin{tabular}{|c|c|c|c|c|}
\hline \multirow[b]{2}{*}{ Subject variable } & \multicolumn{2}{|c|}{ Study 1: Laboratory experiment } & \multicolumn{2}{|c|}{ Study 2: fMRI } \\
\hline & $C G(\mathbb{N}=10)$ & $C C(\mathrm{~N}=57)$ & $C G(\mathrm{~N}=5)$ & $C C(\mathrm{~N}=8)$ \\
\hline Gender (male) (\%) & $5(50 \%)$ & $31(54.39 \%)$ & $2(40 \%)$ & $3(37.5 \%)$ \\
\hline Age (year \pm s.e.) & $37.7(7.30)$ & $37.51(6.48)$ & $38.2(7.12)$ & $38.12(5.96)$ \\
\hline Average years of education ( \pm s.e.) & $11.6(0.97)$ & $12.4(1.91)$ & $11.8(1.09)$ & $12.5(1.60)$ \\
\hline Average years of cocaine use ( \pm s.e.) & $11.5(5.97)$ & $10.05(5.58)$ & $13(3.32)$ & $12.13(6.45)$ \\
\hline Average days of cocaine use/month ( \pm s.e.) & $17.5(10.49)$ & $17.65(8.10)$ & $15.8(11.9)$ & $16(7.8)$ \\
\hline Total amount $(\mathrm{g})$ of cocaine use in the past 90 days ( \pm s.e.) & $29.23(21.42)$ & $61.13(106.2)$ & $20.44(15.65)$ & $56.17(42.08)$ \\
\hline Average years of alcohol use ( \pm s.e.) & $15.89(9.7)$ & $11.19(8.34)$ & $14.75(10.72)$ & $13.12(9.30)$ \\
\hline Average days of alcohol use/month ( \pm s.e.) & $7.9(7.4)$ & $10.55(9.16)$ & $5.2(6.3)$ & $9.25(9.84)$ \\
\hline Regular cigarette smokers: $N(\%)^{*}$ & $10(100 \%)$ & $46(80 \%)$ & $5(100 \%)$ & $6(75 \%)$ \\
\hline
\end{tabular}

Abbreviation: $\mathrm{fMRI}$, functional magnetic resonance imaging.

${ }^{\star} P<0.05$. Note: No significant differences were observed across all variables between the genotype groups in either the laboratory study or the fMRI study of the participants $\left(P^{\prime} \mathrm{S}>0.05\right)$. The CG group included significantly more cigarette smokers than the CC group $(P<0.05)$.

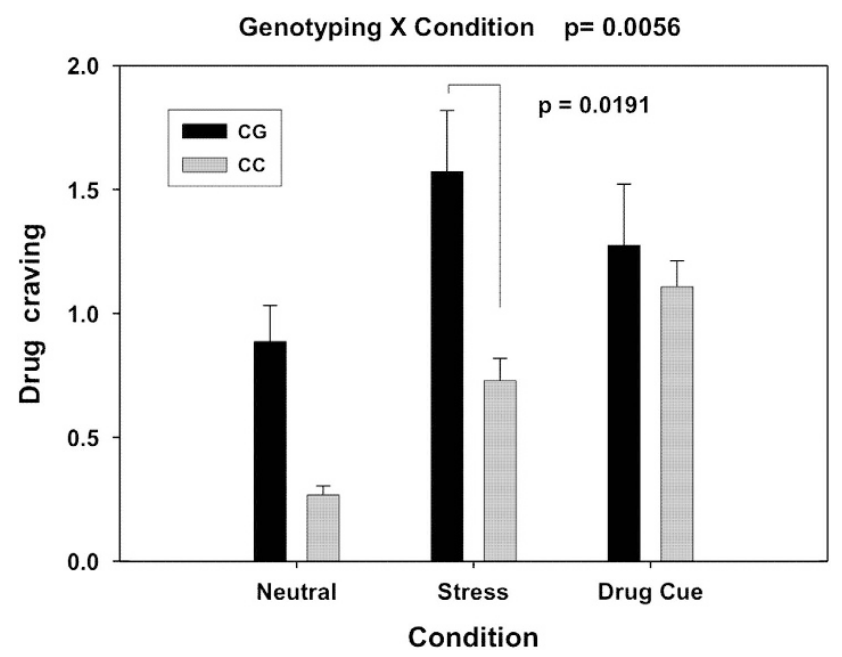

Figure 1 Mean (and standard error) drug craving response (averaged across all time points) during each of the neutral $(\mathrm{N})$, stress $(\mathrm{S})$ and drug cue $(\mathrm{T})$ conditions for the two OPRK1 rs6989250 genotypes is shown. The genotypeby-condition two-way interaction was significant ( $P=0.0056$, Cohen's $d=0.79$ ). Carriers of the $C G$ genotype showed markedly stronger craving when exposed to the stress condition compared with individuals with the CC genotype $(P=0.0191$, Cohen's $d=0.82$ ). There was no significant difference detected between the two genotype groups in either the neutral $(P=0.0823)$ or drug cue conditions $(P=0.678)$.

factor may manifest a marginal main gene effect, we focused on the interactive effect analysis of only rs6989250 with Condition. Consistent with our previous findings, there was a significant effect of Condition on drug craving $(F=20.04$, $\mathrm{df}=2, \quad P<0.001)$. Craving levels were higher under the stress and cue conditions compared with the neutral condition $(P=0.001)$. As mentioned above, there was no main effect of rs6989250 on drug craving after multiple test correction, but there was a significant genotype and condition interaction ( $F=5.40$, df $=2, P=0.006$; effect size Cohen's $d=0.79$ ). Individuals with a CG genotype exhibited a significantly stronger craving than subjects with a $\mathrm{CC}$ genotype during the stress condition $(t=2.370, \mathrm{df}=124$, $P=0.019$, Cohen's $d=0.81)$, but not in the neutral $(P=0.0823)$ and cue conditions $(P=0.678)$ (Figure 1$)$.

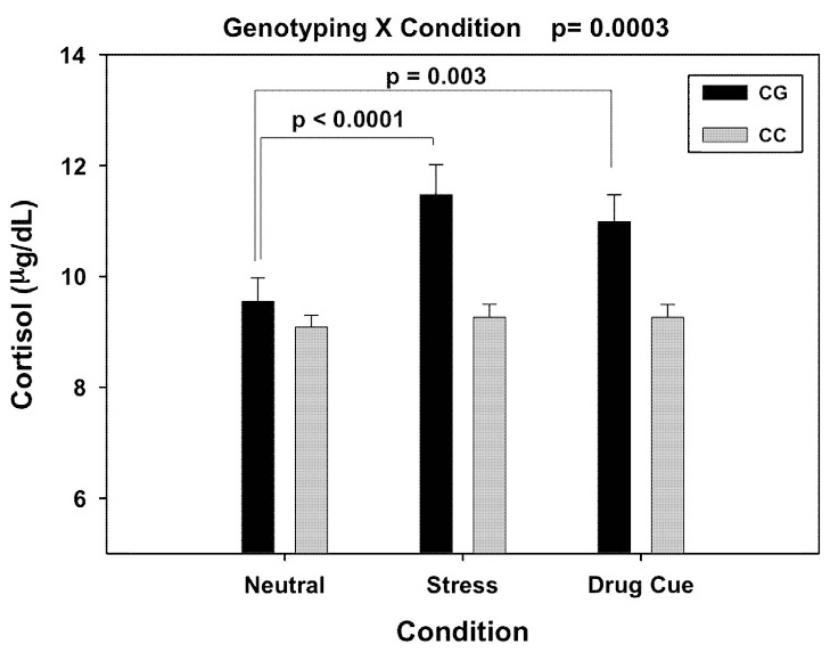

Figure 2 Mean (and standard error) plasma cortisol levels averaged across all time points in the neutral $(\mathrm{N})$, stress $(\mathrm{S})$ and drug cue $(\mathrm{T})$ conditions for the OPRK1 rs6989250 genotype groups are shown. There was a significant genotype-condition interaction ( $P=0.0003$, Cohen's $d=1.01)$, with $C G$ individuals showing higher cortisol levels during stress compared with the neutral and drug cue conditions $(P<0.0001$ for stress versus neutral Cohen's $d=1.46 ; P=0.003$, Cohen's $d=1.04$ for cue versus neutral), whereas CC individuals showed no stress-induced increases in cortisol compared with their cortisol responses in the drug cue and neutral conditions ( $P$ 's $>0.05)$.

Plasma cortisol. Because rs6989250 showed interactive effects with condition, we tested the interaction of rs6989250 with plasma cortisol level. As expected, we found a significant main effect of Condition on plasma cortisol levels ( $F=7.690, \mathrm{df}=2, \quad P=0.0007)$. The cortisol levels were significantly higher during the stress and cue conditions compared with the neutral condition $(t=3.79, \mathrm{df}=2$, $P=0.0002$ for stress versus neutral; $t=2.74, \mathrm{df}=109$, $P=0.0072$ for cue versus neutral). Again, there was no significant main effect of genotype on cortisol levels, but a significant genotype $\times$ condition interaction effect was observed $(F=8.76, \mathrm{df}=2, P=0.0003$ Cohen's $d=1.01)$. The CC group showed no significant increase in cortisol during stress or drug cue exposure, while the CG group showed a highly significant increase in their cortisol 
response during stress $(t=4.25$, df $=109, P<0.0001$, Cohen's $d=1.46)$ and drug cue $(t=3.04, d f=109, P=0.003$, Cohen's $d=1.04$ ) relative to the neutral condition (Figure 2 ).

Cocaine relapse risk. We compared the subsequent cocaine relapse rates during the 90-day follow-up period after discharge from inpatient treatment and research for the $\mathrm{CG}$ and $\mathrm{CC}$ groups. The CG group showed a significantly greater relapse risk than the CC group (Log-rank $\chi^{2}=7.157$; $\mathrm{df}=1 ; P=0.0075$ ) (Figure 3). For example, the abstinence rate in the first 14 days was only $10 \%$ in the CG group but $45 \%$ in the CC group. Thus, individuals with a CG genotype were more likely to relapse and return to cocaine use than the CC group, suggesting that the OPRK1 rs6989250 SNP G allele may increase vulnerability to cocaine relapse.

fMRI results. Whole-brain voxel-wise analysis indicated a significant genotype effect on the brain's response to stress

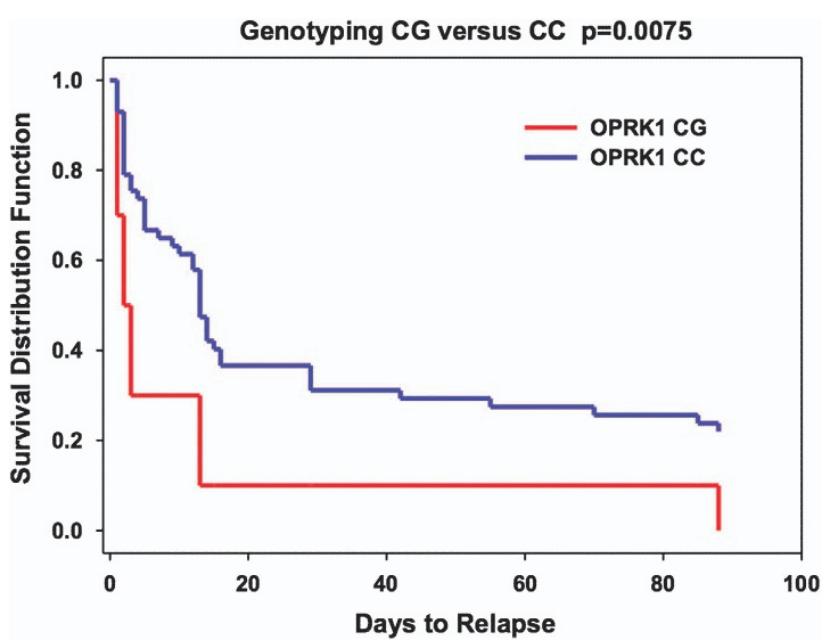

Figure 3 Log-rank survival analysis for abstinence versus cocaine relapse in the $C G$ and $C C$ genotype groups. The survival rate for the $C G$ genotype group was significantly lower than for the CC genotype group (Log-rank $\chi^{2}=5.242$; $P=0.0075)$. Within 14 days, only $10 \%$ individuals with the $C G$ genotype remain abstinence of cocaine use; $45 \%$ individuals with the CC genotype remain abstinence. The $Y$ axis shows the proportion of participants who did not relapse; the $X$ axis shows the 90 -day follow-up time period. and to drug cues relative to neutral cue exposure in cocainedependent subjects $(P<0.05$, whole-brain FWE corrected; Table 2; Figure 4). In both the stress and the drug cues relative to neutral cue contrasts, the CG group showed greater activity in the hypothalamus, midbrain (ventral tegmental area, locus coeruleus), thalamus and cerebellum than the CC group. Additionally, the CG group displayed greater stress-induced activity in the right amygdala/hippocampus and periaqueductal gray area (PAG) of the midbrain than the CC group $(P<0.05$ FWE corrected).

\section{Discussion}

This study showed that the putative functional SNP in the 5 -regulatory region of OPRK1 is associated with significantly higher stress-induced craving, cortisol responses and also increases subsequent risk of cocaine relapse in an AfricanAmerican population with cocaine dependence. In a follow-up study with a subset of subjects, the CG genotype group exhibited stress- and drug cue-induced hyperactivity in hypothalamus/thalamus-midbrain-cerebellum regions, relative to the $\mathrm{CC}$ group. Building on our previous findings identifying clinical and biological factors that are predictive of addiction relapse, ${ }^{1}$ these results support the hypothesis of an interaction between an OPRK1 variant and stress reactivity that results in increased stress-induced cocaine craving, cortisol responses and susceptibility to cocaine relapse in the African-American sample of cocaine-dependent individuals.

Across different drug abusing samples, including cocaine, previous research has shown that exposure to acute stress promotes drug craving and significantly increases the risk of early relapse. ${ }^{47-49}$ Current results indicate that individual variation differed by the OPRK1genotype significantly contributed to the individual variation in the stress-induced craving responses. During laboratory-induced stress exposure, subjective craving was remarkably higher in $C G$ subjects than in CC subjects. In previous research, we have shown that stress-induced drug craving and stress-induced cortisol responses were predictive of earlier relapse to cocaine use, ${ }^{3}$ and thus we expected that such higher stress and drug craving responses in the CG group would make them more susceptible to relapse. Indeed, we find in the current sample that $90 \%$ of the CG subjects relapsed within the first 2 weeks after treatment, suggesting that these

Table 2 Brain regions showing group differences between the CG group and the CC group (CG >CC)

\begin{tabular}{|c|c|c|c|c|c|c|c|c|c|c|c|c|c|}
\hline \multirow[b]{3}{*}{ Regions of activation } & \multirow[b]{3}{*}{ Lat } & \multicolumn{6}{|c|}{ Stress-neutral } & \multicolumn{6}{|c|}{ Drug cue-neutral } \\
\hline & & \multicolumn{3}{|c|}{ Coordinates } & \multirow{2}{*}{$\begin{array}{l}\text { Volume } \\
\left(\mathrm{mm}^{3}\right)\end{array}$} & \multirow[b]{2}{*}{$t$} & \multirow[b]{2}{*}{$d$} & \multicolumn{3}{|c|}{ Coordinates } & \multirow{2}{*}{$\begin{array}{l}\text { Volume } \\
\left(\mathrm{mm}^{3}\right)\end{array}$} & \multirow[b]{2}{*}{$l$} & \multirow[b]{2}{*}{$d$} \\
\hline & & $x$ & $Y$ & $Z$ & & & & $X$ & $Y$ & $Z$ & & & \\
\hline Amygdala/hippocampus & $\mathrm{R}$ & 25 & -7 & -18 & 1226 & 2.31 & 1.32 & - & - & - & - & - & \\
\hline Hypothalamus/thalamus & $B$ & -1 & -5 & -7 & 1735 & 2.39 & 1.36 & 5 & -8 & -6 & 738 & 2.2 & 1.25 \\
\hline Midbrain/brain stem & B & 1 & -26 & -27 & 6925 & 2.38 & 1.36 & 1 & -28 & -27 & 6832 & 2.5 & 1.43 \\
\hline Cerebellum & B & 0 & -39 & -28 & 4823 & 2.46 & 1.40 & 2 & -43 & -27 & 6409 & 2.5 & 1.43 \\
\hline
\end{tabular}

Abbreviations: $\mathrm{B}$, bilateral; $d$, Cohen's d post hoc estimates of effect size; Lat, laterality; $\mathrm{R}$, right.

Note: Significant activations at $P<0.05$ (two-tailed, whole-brain FWE corrected).

Montreal Neurological Institute (MNI) coordinates were used. 
a
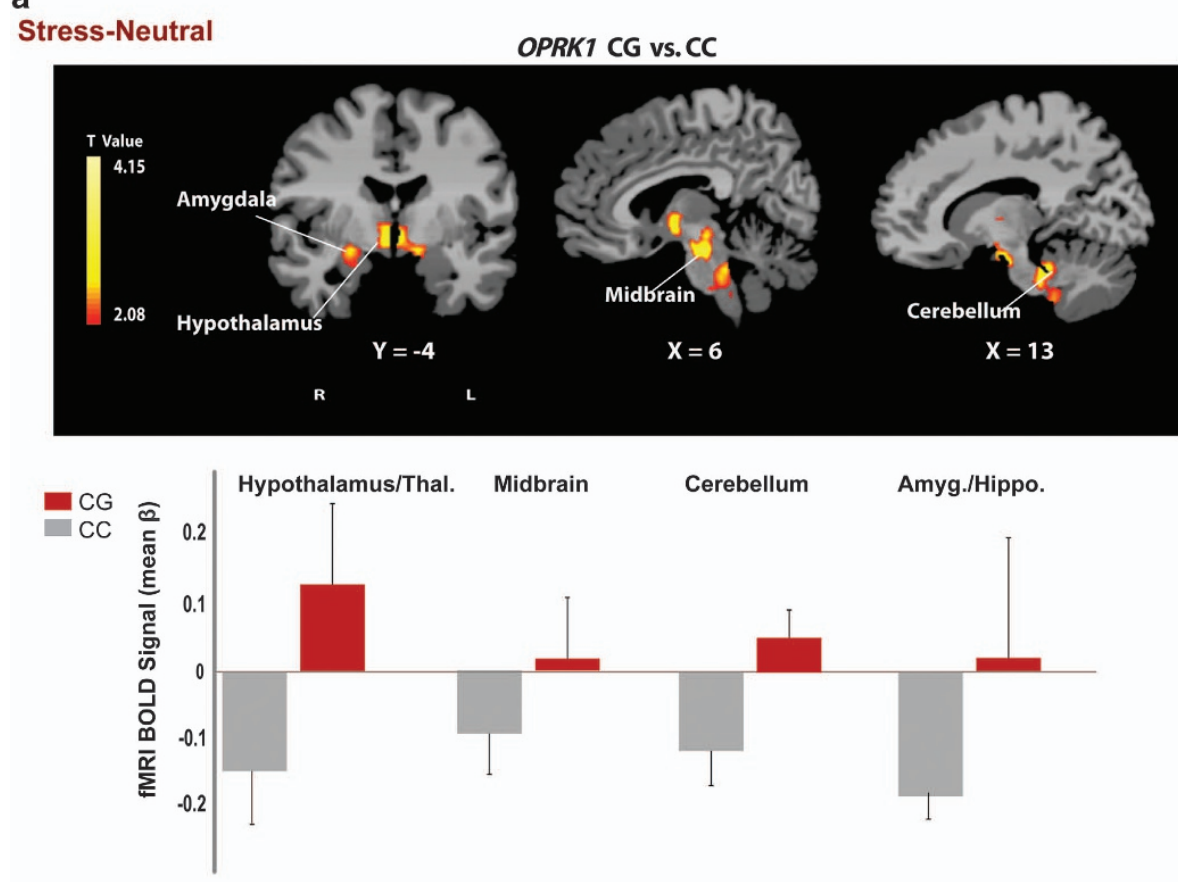

b

Drug-Neutral
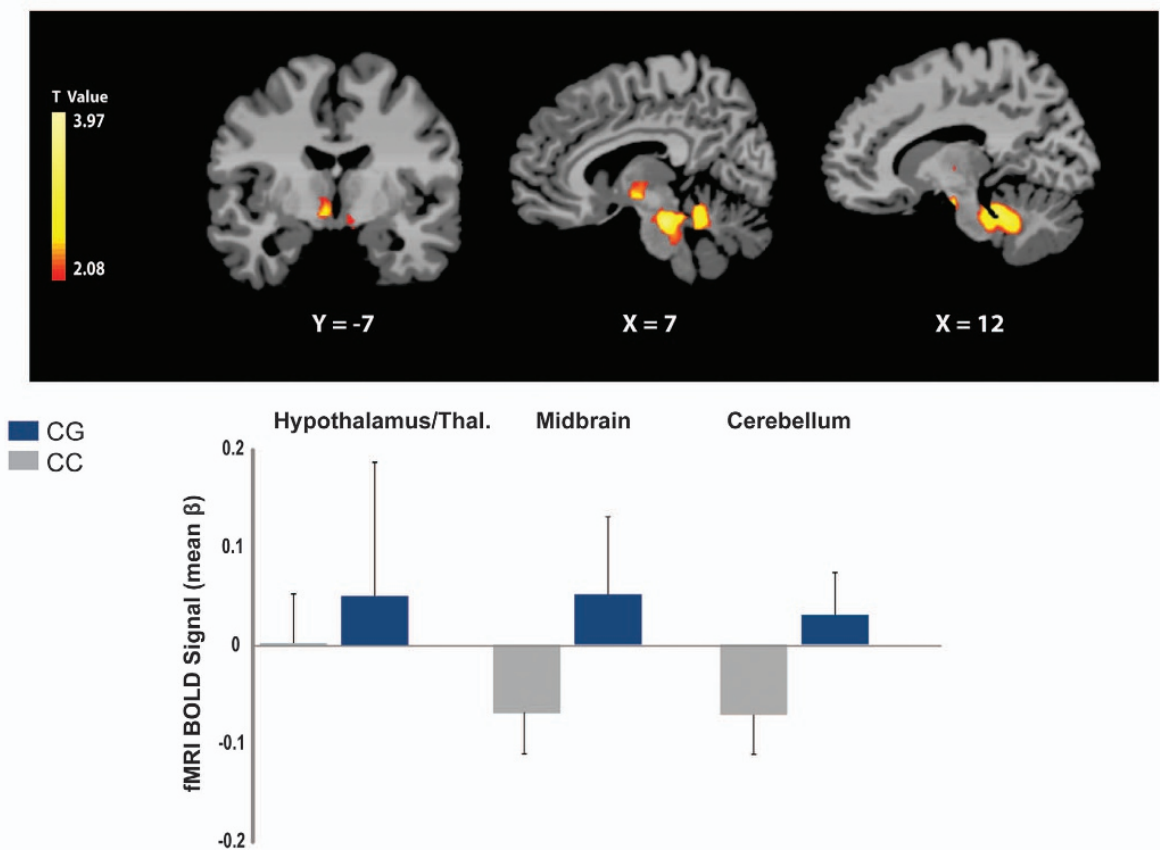

Figure 4 Whole brain analysis of neural activation during (a) stress and (b) drug cue exposure in the CG group relative to the CC group. During the stress and drug cue relative to the neutral condition, the CG group showed increased activity in the hypothalamus, midbrain (ventral tegmental area and locus coeruleus), thalamus and cerebellum relative to the CC group. Additionally, the CG group displayed increased stress-induced activity in the right amygdala/hippocampus and periaqueductal gray (PAG) area of the midbrain compared with the CC group $(P<0.05$, whole-brain family-wise error (FWE) corrected). The blood oxygenation level-dependent (BOLD) functional magnetic resonance imaging (fMRI) signal in each significant cluster (Table 2) is displayed in bar graphs for each genotype group in (a) stress-neutral and (b) drug-neutral conditions (see Table 2 for $t$-values and Cohen's $d$ ). Coordinates are given in Montreal Neurological Institute (MNI) space.

individuals, who showed greater stress-induced cocaine craving, are more vulnerable to an earlier risk of relapse. Interestingly, this effect was specific to the stress condition and was not seen in response to drug cues. These data suggest that the OPRK1 variant specifically affects stress sensitization of drug craving and increases the risk of relapse 
for individuals with this genetic susceptibility. Although these findings were generated from a small sample size, we see medium effect size in the Genotype $\times$ Condition interaction effect for cocaine craving responses.

Consistent with the evidence that KOR modulates stress via the hypothalamic-pituitary-adrenal axis, our results demonstrate that a genetic variant of OPRK1 influences cortisol levels during stress. ${ }^{50,51}$ Previous studies show that KOR agonists increase corticosterone in rats, ${ }^{52}$ elevate plasma cortisol levels in humans ${ }^{53}$ and stimulate plasma ACTH and cortisol release in a dose-dependent manner in non-human primates. ${ }^{54}$ The stimulatory effects of the KOR agonist on the hypothalamic-pituitary-adrenal axis were blocked by the KOR antagonist nor-binaltorphimine. ${ }^{54}$ However, the endogenous kappa opioid peptide dynorphin increases the release of corticotrophin-releasing factor in the hypothalamus. ${ }^{55}$ More importantly, the results of the present study indicate that genetic variation in OPRK1 is associated with individual hypothalamic-pituitary-adrenal responses to stress, as shown by the effect of rs6989250 on the cortisol response in the studied population. Here, we found an interactive effect on cortisol of the rs6989250 genotype and the experimental provocation of stress and drug cue conditions. The cortisol level was significantly higher under stress and drug cue conditions relative to the neutral condition among the subjects with the CG genotype, an effect similar to that seen with a KOR agonist. The genotype effect on cortisol response was large in both stress and drug cue conditions, which is reflected by Cohen's $d>1$. Although the function of rs6989250 is unknown, the $\mathrm{G}$ allele creates an NF-kB transcription factor binding site, presumably resulting in higher OPRK1 expression and increased hypothalamic-pituitary-adrenal axis function. Thus, the current findings suggest that individuals with the $\mathrm{G}$ allele are more vulnerable to stress and stressinduced cortisol response.

The two genotype groups showed significant differences in limbic-midbrain regions, specifically in hypothalamus/ thalamus-midbrain-cerebellum regions during stress and drug cue exposure. The CG group showed greater activity in the hypothalamus, midbrain, thalamus and cerebellum than the CC group during the stress and drug cue relative to the neutral cue trials. KORs are primarily expressed in the hypothalamus and midbrain regions, ${ }^{56}$ consistent with our findings of hyperresponsivity to negative emotion/stress and drug cue challenge in the CG group possibly reflecting an upregulated neuronal function in the regions that are densely packed with KORs. Specifically, the midbrain VTA is important in reward and incentive processing via its involvement in dopamine firing and KOR control of the VTA dopamine projection to the prefrontal cortex. ${ }^{57}$ Additionally, the CG group showed stress-induced hyperactivity in the right amygdala/hippocampus and midbrain PAG. The amygdala and hippocampus have been associated with emotional and stress processing, and hyperactivity in these regions has been reported during exposure to acute and chronic stress. ${ }^{46,58}$ Specifically, right-lateralized limbic activity has been observed during aversive emotional processing, such as in response to fear and negative emotion. ${ }^{59,60}$ The PAG, a midbrain region with abundant connections to the amygdala, is involved in pain, fear and anxiety, ${ }^{61}$ and PAG opioid receptors modulate aversive emotional processing. ${ }^{62}$ The stress-induced hyperactivation observed in the right hypothalamus/thalamus regions and the PAG in the $\mathrm{CG}$ group is consistent with previous findings of association with kappa opioid gene expression related to stress sensitivity and dysphoria. ${ }^{12}$ These results suggest that the neuronal function in limbic-midbrain regions, which are key areas that contain densely distributed KORs, are upregulated during stress and reward processing in CG relative to CC individuals, which could make them more vulnerable to stress and drug craving responses as seen in the laboratory experiment.

It should be noted that genotype effects were of moderate to large effect sizes for each of the dependent measures in this study and larger than those reported for common variants in complex traits. This large genotype effect may be attributable to careful endophenotype characterization of the stress response and minimal population admixture that potentially reduced sample heterogeneity. It is also possible that rs6989250 is indeed functional, or is in linkage disequilibrium with a functional locus on OPRK1.

A limitation of the current findings is the small size of the study sample. Also, the functionality of rs6989250 has not been evaluated. We were unable to perform haplotype analysis because of insufficient statistical power. With these limitations in mind, we suggest caution in interpreting current results. A larger sample size from African-Americans and from other ethnicities and genotyping higher SNP density will be needed in future studies to replicate the results of this study. Further evaluation of the function of the $5^{\prime}$ regulatory variant will also provide more insight into the mechanisms underlying the role of OPRK1 in modulating stress, drug craving and relapse risk associated with cocaine dependence. For example, deep sequencing of the $5^{\prime}$ OPRK1 variant in cocaine-dependent individuals may identify uncommon or rare causal variant(s) of OPRK1.

In summary, to our knowledge, this is the first report of a putative functional SNP in OPRK1 that is linked to individual variation in stress-related drug craving, cortisol and cocaine relapse risk as well as hyperactivation of hypothalamus/thalamus regions during stress and drug cue exposure in cocaine-dependent subjects. Our results indicate a role for $O P R K 1$ in increasing stress, drug craving and cocaine relapse risk in African-Americans. These results also support the need for individualized treatment of cocaine dependence, wherein assessment of genotype and stress vulnerability may help identify those individuals who are most susceptible to early relapse so that development and testing of new, specialized treatments may be considered for such individuals.

\section{Conflict of interest}

Dr. Sinha is on the Scientific Advisory Board for Embera Neurotherapeutics. The remaining authors declare no conflict of interest.

Acknowledgements. This study was supported by the following National Institute on Drug Abuse grants: P50-DA016556 (RS) and the (K12DA000167) (MP) and the APA/Merck Early Career Development Award and by the Department of Mental Health and Addiction Services of the State of Connecticut. We also thank the 
staff at the Clinical Neuroscience Research Unit of the Connecticut Mental Health Center, the Yale Stress Center and the Yale Magnetic Resonance Research Center (MRRC) for their contribution to this project.

1. Sinha R. New findings on biological factors predicting addiction relapse vulnerability. Cur Psychiatry Rep 2011; 13: 398-405.

2. Sinha R, Fox HC, Hong KA, Bergquist K, Bhagwagar Z, Siedlarz KM. Enhanced negative emotion and alcohol craving, and altered physiological responses following stress and cue exposure in alcohol dependent individuals. Neuropsychopharmacology 2009; 34 : 1198-1208.

3. Sinha R, Garcia M, Paliwal P, Kreek MJ, Rounsaville BJ. Stress-induced cocaine craving and hypothalamic-pituitary-adrenal responses are predictive of cocaine relapse outcomes. Arch Gen Psychiatry 2006; 63: 324-331.

4. Bruchas MR, Land BB, Chavkin C. The dynorphin/kappa opioid system as a modulator of stress-induced and pro-addictive behaviors. Brain Res 1314: 44-55.

5. Wadenberg ML. A review of the properties of spiradoline: a potent and selective kappaopioid receptor agonist. CNS Drug Rev 2003; 9: 187-198.

6. Pfeiffer A, Brantl V, Herz A, Emrich HM. Psychotomimesis mediated by kappa opiate receptors. Science 1986; 233: 774-776.

7. Bals-Kubik R, Ableitner A, Herz A, Shippenberg TS. Neuroanatomical sites mediating the motivational effects of opioids as mapped by the conditioned place preference paradigm in rats. The J Pharmacol Exp Ther 1993; 264: 489-495.

8. Butelman ER, Yuferov V, Kreek MJ. kappa-opioid receptor/dynorphin system: genetic and pharmacotherapeutic implications for addiction. Trends Neurosci 2012; 35: 587-596.

9. Mague SD, Pliakas AM, Todtenkopf MS, Tomasiewicz HC, Zhang Y, Stevens WC Jr. et al. Antidepressant-like effects of kappa-opioid receptor antagonists in the forced swim test in rats. J Pharmacol Exp Ther 2003; 305: 323-330.

10. Carlezon WA Jr., Beguin C, DiNieri JA, Baumann MH, Richards MR, Todtenkopf MS et al. Depressive-like effects of the kappa-opioid receptor agonist salvinorin $A$ on behavior and neurochemistry in rats. J Pharmacol Exp Ther 2006; 316: 440-447.

11. Przewlocki R, Lason W, Hollt V, Silberring J, Herz A. The influence of chronic stress on multiple opioid peptide systems in the rat: pronounced effects upon dynorphin in spinal cord. Brain Res 1987; 413: 213-219.

12. Land BB, Bruchas MR, Lemos JC, Xu M, Melief EJ, Chavkin C. The dysphoric component of stress is encoded by activation of the dynorphin kappa-opioid system. J Neurosci 2008; 28: 407-414.

13. Bilkei-Gorzo A, Racz I, Michel K, Mauer D, Zimmer A, Klingmuller D et al. Control of hormonal stress reactivity by the endogenous opioid system. Psychoneuroendocrinology 2008; 33: 425-436.

14. McLaughlin JP, Li S, Valdez J, Chavkin TA, Chavkin C. Social defeat stress-induced behavioral responses are mediated by the endogenous kappa opioid system. Neuropsychopharmacology 2006; 31: 1241-1248.

15. Koob GF. A role for brain stress systems in addiction. Neuron 2008; 59: 11-34.

16. McLaughlin JP, Land BB, Li S, Pintar JE, Chavkin C. Prior activation of kappa opioid receptors by $U 50,488$ mimics repeated forced swim stress to potentiate cocaine place preference conditioning. Neuropsychopharmacology 2006; 31: 787-794.

17. Fox HC, Talih M, Malison R, Anderson GM, Kreek MJ, Sinha R. Frequency of recent cocaine and alcohol use affects drug craving and associated responses to stress and drug-related cues. Psychoneuroendocrinology 2005; 30: 880-891.

18. Hyman SM, Fox H, Hong KI, Doebrick C, Sinha R. Stress and drug-cue-induced craving in opioid-dependent individuals in naltrexone treatment. Exp Clin Psychopharmacol 2007; 15: 134-143.

19. Breese GR, Chu K, Dayas CV, Funk D, Knapp DJ, Koob GF et al. Stress enhancement of craving during sobriety: a risk for relapse. Alcoholism Clin Exp Res 2005; 29: 185-195.

20. Sinha R, Fuse T, Aubin LR, O'Malley SS. Psychological stress, drug-related cues and cocaine craving. Psychopharmacology 2000; 152: 140-148.

21. Karpyak VM, Winham SJ, Preuss UW, Zill P, Cunningham JM, Walker DL et al. Association of the PDYN gene with alcohol dependence and the propensity to drink in negative emotional states. Int J Neuropsychopharmacol 2013; 16: 975-985.

22. Wee S, Koob GF. The role of the dynorphin-kappa opioid system in the reinforcing effects of drugs of abuse. Psychopharmacology 2010; 210: 121-135.

23. Ungless MA, Argilli E, Bonci A. Effects of stress and aversion on dopamine neurons: implications for addiction. Neurosci Biobehav Rev 2010; 35: 151-156.

24. Hurd YL, Herkenham M. Molecular alterations in the neostriatum of human cocaine addicts. Synapse 1993; 13: 357-369.

25. Xuei X, Dick D, Flury-Wetherill L, Tian HJ, Agrawal A, Bierut $L$ et al. Association of the kappa-opioid system with alcohol dependence. Mol Psychiatry 2006; 11: 1016-1024.

26. Zhang H, Kranzler HR, Yang BZ, Luo X, Gelernter J. The OPRD1 and OPRK1 loci in alcohol or drug dependence: OPRD1 variation modulates substance dependence risk. Mol Psychiatry 2008; 13: 531-543.

27. Gerra G, Leonardi C, Cortese E, D'Amore A, Lucchini A, Strepparola G et al. Human kappa opioid receptor gene (OPRK1) polymorphism is associated with opiate addiction. Am J Med Genet B Neuropsychiatr Genet 2007; 144B: 771-775.
28. Gelernter J, Gueorguieva R, Kranzler HR, Zhang H, Cramer J, Rosenheck R et al. Opioid receptor gene (OPRM1, OPRK1, and OPRD1) variants and response to naltrexone treatment for alcohol dependence: results from the VA Cooperative Study. Alcoholism Clin Exp Res 2007; 31: 555-563.

29. Loh el W, Fann CS, Chang YT, Chang CJ, Cheng AT. Endogenous opioid receptor genes and alcohol dependence among Taiwanese Han. Alcoholism Clin Exp Res 2004; 28: 15-19.

30. Ashenhurst JR, Bujarski S, Ray LA. Delta and kappa opioid receptor polymorphisms influence the effects of naltrexone on subjective responses to alcohol. Pharmacol Biochem Behav 2012; 103: 253-259.

31. Saito M, Ehringer MA, Toth R, Oros M, Szakall I, Sikela JM et al. Variants of kappa-opioid receptor gene and mRNA in alcohol-preferring and alcohol-avoiding mice. Alcohol 2003; 29: 39-49.

32. Edenberg HJ, Wang J, Tian $H$, Pochareddy S, Xuei X, Wetherill $L$ et al. A regulatory variation in OPRK1, the gene encoding the kappa-opioid receptor, is associated with alcohol dependence. Hum Mol Genet 2008; 17: 1783-1789.

33. First MB, Spitzer RL, Gibbon M, Williams JBW. Structured Clinical Interview for DSM-IV (Patient Edition). American Psychiatric Press Inc: Washington DC, 1995; 1995.

34. Sinha R. Modeling stress and drug craving in the laboratory: implications for addiction treatment development. Addict Biol 2009; 14: 84-98.

35. Sinha R, Talih M, Malison R, Cooney N, Anderson GM, Kreek MJ. Hypothalamic-pituitaryadrenal axis and sympatho-adreno-medullary responses during stress-induced and drug cue-induced cocaine craving states. Psychopharmacology 2003; 170: 62-72.

36. Sinha R, Lacadie C, Skudlarski P, Fulbright RK, Rounsaville BJ, Kosten TR et al. Neural activity associated with stress-induced cocaine craving: a functional magnetic resonance imaging study. Psychopharmacology 2005; 183: 171-180.

37. Sinha R. Modeling relapse situations in the human laboratory. Curr Top Behav Neurosci 2011.

38. Hodgkinson CA, Yuan Q, Xu K, Shen PH, Heinz E, Lobos EA et al. Addictions biology: haplotype-based analysis for 130 candidate genes on a single array. Alcohol Alcohol 2008; 43: 505-515.

39. Pritchard JK, Stephens M, Donnelly P. Inference of population structure using multilocus genotype data. Genetics 2000; 155: 945-959.

40. Hommer RE, Seo D, Lacadie CM, Chaplin TM, Mayes LC, Sinha R et al. Neural correlates of stress and favorite-food cue exposure in adolescents: a functional magnetic resonance imaging study. Hum Brain Mapp advance online publication, 16 April 2012; doi: 10.1002/ hbm.22089 (e-pub ahead of print).

41. Nolfe E. XMedCon- An open-source medical image conversion toolkit. Eur J Nucl Med 2003; 30(Suppl 2): S246.

42. Friston KJ, Holmes A, Poline JB, Price CJ, Frith CD. Detecting activations in PET and fMRI: levels of inference and power. Neuroimage 1996; 4(3 Pt 1): 223-235.

43. Duncan JS, Papademetris X, Yang J, Jackowski M, Zeng X, Staib LH. Geometric strategies for neuroanatomic analysis from MRI. Neuroimage 2004; 23(Suppl 1): S34-S45.

44. Holmes CJ, Hoge R, Collins L, Woods R, Toga AW, Evans AC. Enhancement of MR images using registration for signal averaging. J Comput Assist Tomogr 1998; 22: 324-333.

45. Cox RW. AFNl: software for analysis and visualization of functional magnetic resonance neuroimages. Comput Biomed Res 1996; 29: 162-173.

46. Seo D, Jia Z, Lacadie CM, Tsou KA, Bergquist K, Sinha R. Sex differences in neural responses to stress and alcohol context cues. Human Brain Mapping 2011; 32: 1998-2013.

47. Sinha R, Fox HC, Hong KI, Hansen J, Tuit K, Kreek MJ. Effects of adrenal sensitivity, stress- and cue-induced craving, and anxiety on subsequent alcohol relapse and treatment outcomes. Arch Gen Psychiatry 2011; 68: 942-952.

48. Sinha R. How does stress increase risk of drug abuse and relapse? Psychopharmacology 2001; 158: 343-359.

49. Sinha R. Chronic stress, drug use, and vulnerability to addiction. Ann NY Acad Sci 2008; 1141: $105-130$.

50. Buckingham JC, Cooper TA. Pharmacological characterization of opioid receptors influencing the secretion of corticotrophin releasing factor in the rat. Neuroendocrinology 1986; 44: 36-40.

51. Nikolarakis K, Pfeiffer A, Stalla GK, Herz A. The role of CRF in the release of ACTH by opiate agonists and antagonists in rats. Brain Res 1987; 421: 373-376.

52. Laorden ML, Milanes MV. Effects of U- $50488 \mathrm{H}$ and U-50 $488 \mathrm{H}$ withdrawal on catecholaminergic neurons of the rat hypothalamus. Life Sci 2000; 66: 803-815.

53. Ur E, Wright DM, Bouloux PM, Grossman A. The effects of spiradoline (U-62066E), a kappa-opioid receptor agonist, on neuroendocrine function in man. Br J Pharmacol 1997; 120: 781-784.

54. Pascoe JE, Williams KL, Mukhopadhyay P, Rice KC, Woods JH, Ko MC. Effects of mu, kappa, and delta opioid receptor agonists on the function of hypothalamic-pituitary-adrenal axis in monkeys. Psychoneuroendocrinology 2008; 33: 478-486.

55. Szeto HH. Dynorphin and the hypothalamo-pituitary-adrenal axis during fetal development. Life Sci 2003; 73: 749-758.

56. Mansour A, Fox CA, Akil H, Watson SJ. Opioid-receptor mRNA expression in the rat CNS: anatomical and functional implications. Trends Neurosci 1995; 18: 22-29.

57. Margolis EB, Lock H, Chefer VI, Shippenberg TS, Hjelmstad GO, Fields HL. Kappa opioids selectively control dopaminergic neurons projecting to the prefrontal cortex. Proc Natl Acad Sci USA 2006; 103: 2938-2942. 
58. Ganzel B, Casey BJ, Glover G, Voss HU, Temple E. The aftermath of 9/11: effect of intensity and recency of trauma on outcome. Emotion 2007; 7: 227-238.

59. Morris JS, Ohman A, Dolan RJ. A subcortical pathway to the right amygdala mediating 'unseen' fear. Proc Natl Acad Sci USA 1999; 96: 1680-1685.

60. Lanteaume L, Khalfa S, Regis J, Marquis P, Chauvel P, Bartolomei F. Emotion induction after direct intracerebral stimulations of human amygdala. Cereb Cortex 2007; 17 1307-1313.

61. Behbehani MM. Functional characteristics of the midbrain periaqueductal gray. Prog Neurobiol 1995; 46: 575-605.
62. McNally GP, Pigg M, Weidemann G. Opioid receptors in the midbrain periaqueductal gray regulate extinction of pavlovian fear conditioning. J Neurosci 2004; 24: 6912-6919.

(c) (i) (s) $\odot$ Translational Psychiatry is an open-access journal (1) ${ }_{\mathrm{BY}} \mathrm{ND}$ published by Nature Publishing Group. This work is licensed under the Creative Commons Attribution-NonCommercial-No Derivative Works 3.0 Unported License. To view a copy of this license, visit http://creativecommons.org/licenses/by-nc-nd/3.0/

Supplementary Information accompanies the paper on the Translational Psychiatry website (http://www.nature.com/tp) 